\title{
Ajuste de equações hipsométricas para a estimação da altura total de indivíduos jovens de teca
}

\author{
Hypsometric equations adjustment to estimate the total height of young trees \\ of teak
}

\begin{abstract}
Mateus Niroh Inoue SANQUETTA ${ }^{1}$; Carlos Roberto SANQUETTA ${ }^{2}$; Francelo MOGNON ${ }^{3}$; Ana Paula Dalla CORTE ${ }^{2}$; Aurélio Lourenço RODRIGUES ${ }^{3}$; Greyce Charllyne Benedet MAAS ${ }^{3}$

${ }^{1}$ Autor para correspondência - Discente em Engenharia Florestal, Universidade Federal do Paraná, Av. Lothário Meissner, 900, Jardim Botânico, Curitiba, PR, mateus.sanquetta@hotmail.com

${ }^{2}$ Engenheiro Florestal, Dr., Professor do Departamento de Ciências Florestais, Universidade Federal do Paraná, carlos_sanquetta@hotmail.com; anapaulacorte@gmail.com

${ }^{3}$ Discente do Programa de Pós-Graduação em Engenharia Florestal, Universidade Federal do Paraná, mognonf@gmail.com; alourencorodrigues@gmail.com; greycemaas@yahoo.com.br
\end{abstract}

\section{Resumo}

Recebido em: 03-12-2014; Aceito em: 06-05-2015

A teca (Tectona grandis L. f.) possui uma das madeiras mais valiosas do mundo, e por este motivo é alvo de muitos estudos. Em razão de o início de seu cultivo ser recente no Brasil, são poucos os estudos relativos à modelagem biométrica de teca no País. O presente trabalho teve como objetivo testar seis diferentes modelos hipsométricos para estimar a altura total em 400 indivíduos juvenis de teca na região de Redenção-PA, medidos com hipsômetro Vértex IV. Para tanto, foram utilizados quatro diferentes critérios de seleção: coeficiente de determinação ajustado ( $\mathrm{R}^{2} \mathrm{aj}$. ), erro-padrão da estimativa (Syx\%), critério de informação de Akaike (AIC) e critério de informação Bayesiano (BIC), sendo a análise da distribuição dos resíduos também empregada para esse fim. Trinta e cinco árvores foram derrubadas para medição real de suas alturas, as quais foram empregadas para validação da melhor equação. Os indivíduos utilizados neste estudo apresentaram diâmetro a altura do peito (DAP) médio de $11,07 \pm 0,42 \mathrm{~cm}$ e altura total média (ht) de $9,25 \pm 0,31 \mathrm{~m}$. Os seis modelos mostraram indicadores gerais de ajuste satisfatórios, mas a análise de resíduos evidenciou que quatro deles apresentam vieses nas estimativas. $O$ modelo de Trorey foi selecionado como o mais preciso e acurado, considerando os critérios gerais de ajuste e a análise de resíduos, com $\mathrm{R}^{2}$ aj. de 0,89, Syx\% de 11,37\%, AIC de 46,16 e 50,04 de BIC. A equação gerada pelo modelo de Trorey foi validada com dados independentes, proporcionando estimativas confiáveis e sem viés, considerando as condições vigentes neste estudo.

Palavras-chave adicionais: critérios de seleção; dendrometria; inventário florestal; manejo florestal; modelagem; Tectona grandis.

\begin{abstract}
Teak (Tectona grandis $L$. f.) is one of the most valuable timbers in the world and, therefore, has been subject of various studies. However, a few modeling studies on biometrical measures of this species have been carried out so far in Brazil because this cultivation is still very recent in the country. This study aimed to test six different hypsometric models to estimate the total height of 400 young teak individuals in the region of Redenção-PA, measured with the Vertex IV hypsometer. Four different selection criteria were used: adjusted coefficient of determination $\left(\mathrm{R}^{2}\right.$ ai.), standard error of estimate $\left(\mathrm{S}_{\mathrm{yx}} \%\right)$, Akaike information criteria (AIC) and Bayesian information criteria (BIC), and the graphical analysis of residuals was also performed. Thirty-five trees were felled and their actual heights were directly measured using tape, which were used to validate the best fitted equation. The tress used in this study had a mean of diameter at breast height (DAP) of $11.07 \pm 0.42 \mathrm{~cm}$ and total height (ht) of $9.25 \pm 0.31 \mathrm{~m}$. The six models showed overall satisfactory indicators of fitting, but the graphical analysis of residuals showed that four of them produce biased estimates. The Trorey model was elected as been the most precise and accurate, considering the general criteria of fit and the graphical residual analysis of wastes, with $\mathrm{R}^{2}$ aj. of $0.89, \mathrm{~S}_{\mathrm{y}} \%$ of $11.37 \%$, and 46.16 and 50.04 of AIC and $\mathrm{BIC}$, respectively. The equation generated by the Trorey model was validated using independent data and demonstrated providing reliable and unbiased estimates, considering the conditions prevailing in this study.
\end{abstract}

Additional keywords: forest inventory; forest management; modeling; selection criteria; Tectona grandis; tree measurement.

\section{Introdução}

A madeira da teca (Tectona grandis L. f.) é conhecida mundialmente por sua beleza e durabilidade, fatos que the agregam alto valor comercial (Perez Cordero \& Kanninen, 2003). A demanda mundial por esse produto fez com que países onde essa espécie não é nativa adotassem seu cultivo (Hoare \& Patanapongsa, 1988; Monteuuis \& Goh,
1999; Bhat, 2000), como a Costa Rica em 1940 (Keogh et al., 1978), e mais recentemente o Brasil, onde, segundo a ABRAF (2012), existem, aproximadamente, 67 mil hectares com plantios distribuídos nas regiões Norte e Centro-Oeste.

De acordo com levantamentos da FAO (2012), existem cerca de 4,6 milhões de hectares de plantações de teca no mundo, tendo como base o ano de 2010. No entanto, disponibilidade dessa fonte 
de matéria-prima está abaixo do necessário. Apesar de a teca ser considerada uma espécie de rápido crescimento (Leite et al., 2011), em condições para plantio comercial, a qualidade de sua madeira e a produtividade podem ser inferiores ao esperado (Tze, 1999). Por esse motivo, pouco se sabe a respeito da produtividade de plantios comerciais de teca no Brasil.

Sanquetta et al. (2014a) concluíram que, na região de Redenção-PA, os plantios de teca apresentaram ótimo desempenho na fase inicial de crescimento. No entanto, para avaliar o crescimento e a produtividade de um plantio florestal, é necessário realizar 0 inventário florestal. Basicamente, o inventário florestal consiste na medição de variáveis como Altura (ht), Diâmetro à Altura do Peito (DAP) e, consequentemente, na estimativa do volume e outras grandezas de interesse comercial. Por ser uma variável de difícil obtenção quando comparada ao diâmetro, a altura é muitas vezes mensurada em um número menor de árvores e, posteriormente, utiliza-se de estimativa por meio de modelos de regressões lineares, conhecida como relação hipsométrica.

Muito usado no meio florestal, o ajuste de equações hipsométricas é de grande importância para a estimação do volume. Diversos pesquisadores empenham-se no sentido de desenvolver modelos para a estimação de diferentes variáveis, neste caso a altura. Este método proporciona a estimativa da altura total da árvore a partir de outra variável de mais fácil obtenção, como, por exemplo, o DAP. A relação hipsométrica, conforme Schmidt (1977), pode ser definida como a regressão da altura da árvore pelo seu respectivo diâmetro. Por meio de regressão linear, modelos matemáticos são testados, e equações são ajustadas para a obtenção das estimativas.

No entanto, devido às variações morfológicas das espécies, alguns modelos tendem a subestimar ou superestimar a variável de interesse. No Brasil, são poucos os trabalhos no tocante à relação hipsométrica em plantios de teca. Desta forma, o objetivo deste estudo foi avaliar o comportamento de diferentes modelos hipsométricos para estimar a altura total de indivíduos da espécie Tectona grandis oriundo de plantios na região de Redenção-PA. Sendo assim, a seguinte hipótese foi elaborada para ser testada neste trabalho: "A Altura total de indivíduos jovens de teca apresenta relação direta com o Diâmetro à Altura do Peito, sendo possível o desenvolvimento de equações hipsométricas satisfatórias para plantios jovens da espécie".

\section{Material e métodos}

O presente estudo foi desenvolvido com dados advindos de plantios florestais localizados na região de Redenção, no sudeste do Estado do Pará. Segundo a classificação de Köppen, o clima da região é o equatorial superúmido (Am) no limite de transição para Aw. A temperatura média anual é de $25^{\circ} \mathrm{C}$, com máxima e mínima de $32^{\circ} \mathrm{C}$ e $22^{\circ} \mathrm{C}$, respectivamente, e a umidade relativa do ar é alta durante todo o ano, em média 78\% (IDESP, 2012).

Os indivíduos dos plantios em questão são jovens, com idades entre 4 e 5 anos, e foram estabelecidos em povoamentos puros, de espaçamento $4 \times 4 \mathrm{~m}$. Durante o mês de dezembro de 2012, foi realizado um inventário florestal no qual foram medidos todos os diâmetros à altura do peito dos indivíduos. Foram medidas as Alturas totais (ht) de aproximadamente 10 a $20 \%$ das árvores dos plantios, utilizando hipsômetro Vértex IV. Para este estudo, foram utilizados 400 pares de valores de DAP e ht. Na ocasião, 35 árvores foram derrubadas e tiveram suas alturas reais mensuradas rigorosamente com trena. Os dados dendrométricos obtidos em campo foram tabulados e aplicou-se a estatística descritiva convencional, calculando-se as respectivas medidas de tendência central (média) e dispersão (desvio-padrão). Posteriormente, aplicou-se o teste de correlação de Pearson com o objetivo de verificar a correlação entre as variáveis DAP e ht.

Foram testados seis modelos hipsométricos, sendo utilizada como variável dependente ht e como variável independente o DAP (Tabela 1). Aplicou-se a técnica da regressão linear pelo método dos mínimos quadrados ordinários aos seis modelos testados.

Tabela 1 - Modelos hipsométricos testados para 400 indivíduos jovens de Tectona grandis em Redenção-PA. Hypsometric models tested for 400 young individuals of Tectona grandis in Redenção-PA.

\begin{tabular}{lll}
\hline & \multicolumn{1}{c}{ Modelo } & \multicolumn{1}{c}{ Autor(es) } \\
\hline$(1)$ & $h t=\beta_{0}+\beta_{1} \cdot$ DAP & Linear Simples \\
$(2)$ & $h t=\beta_{0}+\beta_{1} \cdot \mathrm{DAP}^{2}$ & Azevedo et al. (1999) \\
$(3)$ & $h t=\beta_{0}+\beta_{1} \cdot \ln (\mathrm{DAP})$ & Henricksen (1950) \\
$(4)$ & $h t=\beta_{0}+\beta_{1} \cdot \mathrm{DAP}+\beta_{2} . \mathrm{DAP}{ }^{2}$ & Trorey (1932) \\
$(5)$ & $\ln (h t)=\beta_{0}+\beta_{1} \cdot(1 / \mathrm{DAP})$ & Curtis (1967) \\
$(6)$ & $\ln (h t)=\beta_{0}+\beta_{1} \cdot \ln (\mathrm{DAP})$ & Stoffels \& Soest (1953) \\
\hline
\end{tabular}

$\beta_{0}, \beta_{1}$ e $\beta_{2}$ - coeficientes estimados por regressão; DAP - diâmetro à altura do peito $(\mathrm{cm})$; ht - altura total do fuste (m); In- logaritmo natural.

Para os modelos logarítmicos, os valores estimados foram obtidos por antilogaritmização natural seguida de aplicação do Fator de Correção de Meyer (FCM):

$F C M=e^{0,5 S y x^{2}}$

\section{Em que:}

e - base dos logaritmos naturais $=2,718281828$;

Syx - erro padrão da estimativa

Os seis modelos testados foram avaliados 
segundo quatro critérios gerais de qualidade de ajuste e pela análise da distribuição dos resíduos. Os critérios utilizados são os seguintes:

1) Coeficiente de determinação ajustado $\left(R^{2}\right.$ aj. $)$ :

$$
\mathrm{R}^{2} \text { aj. }=1-\frac{(\mathrm{n}-1)}{(\mathrm{n}-\mathrm{k})}\left(1-\mathrm{R}^{2}\right)
$$

Em que:

$\mathrm{n}$ - número de observações;

k - número de parâmetros do modelo;

$\mathrm{R}^{2}$ - coeficiente de determinação do modelo

$$
R^{2}=1 \frac{\sum_{i=1}^{n} e_{i}^{2}}{\sum_{i=1}^{n}\left(h t_{i}-\bar{h} t\right)^{2}}
$$

e

$$
\sum_{i=1}^{n} e_{i}^{2}=\sum_{i=1}^{n}\left(h t_{i}-\hat{h} t_{i}\right)^{2}
$$

2) Erro-padrão da estimativa, em porcentagem:

$$
\text { Syx } \%=\frac{\text { Syx }}{\bar{h} t} * 100
$$

Em que:

Syx - erro-padrão da estimativa absoluto (m); ht- altura total (m).

e

$$
S_{y x}=\sqrt{\frac{\sum_{i 1}^{n}\left(h t_{i}-\hat{h} t_{i}\right)^{2}}{n-k}}
$$

3) Critério de Informação de Akaike não inviesado para pequenas amostras ${ }^{*}$, usado quando $\frac{n}{k}<40$ (AKAIKE, 1973):

$$
\mathrm{AIC}_{\mathrm{C}}=-2\left(\frac{-\mathrm{n}}{2} \ln \left(\frac{1}{\mathrm{n}} \sum_{\mathrm{i}=1}^{\mathrm{n}} \mathrm{e}_{\mathrm{i}}^{2}\right)\right)+2 \mathrm{k} \frac{\mathrm{n}}{(\mathrm{n}-\mathrm{k}-1)}
$$

4) Critério de Informação de Bayesiano ou de Schwarz (1978):

$$
\mathrm{BIC}_{\mathrm{c}}=-2\left(\frac{-\mathrm{n}}{2} \ln \left(\frac{1}{\mathrm{n}} \sum_{\mathrm{i}=1}^{\mathrm{n}} \mathrm{e}_{\mathrm{i}}^{2}\right)\right)+\ln (\mathrm{n}) \mathrm{k}
$$

* Segundo Burnham \& Anderson (2002). Em que: $\mathrm{n}$ - número de observações; $\mathrm{k}$ - número de parâmetros do modelo + 1 (intercepto); ei2 - erro associado; In - logaritmo natural.

Em $\mathrm{AIC}_{c}$ e BIC, o valor de $k$ deve ser acrescido de 1 no número de coeficientes do modelo, que se refere a um grau de liberdade da variância.

A análise dos resíduos foi realizada por meio dos gráficos dos resíduos em percentagem, ou seja:

$$
\text { resíduo\% }=\frac{\mathrm{ht}_{\mathrm{i}}-\hat{\mathrm{ht}}_{\mathrm{i}}}{\mathrm{ht}_{\mathrm{i}}} * 100
$$

Foi realizada a validação do melhor modelo testado por meio dos testes de Qui-quadrado e de médias t-pareado, ao nível de $95 \%$ de confiança.

\section{Resultados e discussão}

Os indivíduos avaliados no presente trabaIho atingiram, em média, 11,07 cm de DAP e 9,25 m de ht. As medidas de dispersão (desvio-padrão) foram $4,27 \mathrm{~cm}$ e $3,20 \mathrm{~m}$ para as duas variáveis, respectivamente, significando erros de amostragem de $0,42 \mathrm{~cm}$ e $0,31 \mathrm{~m}$, respectivamente, ante uma intensidade amostral de 400 observações.

Os valores de DAP e ht retrocitados demonstram o grande potencial de crescimento da espécie. Sanquetta et al. (2014a) destacam o ótimo crescimento inicial de Tectona grandis na região deste estudo. No que concerne às variáveis dendrométricas de teca, outros autores observaram valores inferiores aos aqui encontrados. Christo et al. (2012), estudando indivíduos de teca com cinco anos de idade, na região de Alta Floresta, no Estado do Mato Grosso, observaram DAP médio de $12,62 \mathrm{~cm}$ e ht média de $12,66 \mathrm{~m}$. Tonini et al. (2009), ao avaliarem o crescimento inicial de plantios de teca no Estado de Roraima, observaram que, aos quatro e cinco anos, os plantios apresentaram DAP de 5,39 e 6,61 cm e ht de 8,91 e $10,29 \mathrm{~m}$, respectivamente. Isso mostra o potencial da região de Redenção em comparação a outras do País no que concerne ao desempenho da espécie.

Observou-se uma relação direta entre DAP e ht. Essa relação foi significativa $(\alpha=0,05$,$) conside-$ rando indivíduos com diâmetro variando entre 2,20 e $22,20 \mathrm{~cm}$ e altura entre 1,40 e 17,1 m. Este fato pode ser confirmado pelo alto coeficiente de correlação de Pearson entre as variáveis $(r=0,94)$.

A partir dos modelos testados, ajustaram-se as respectivas equações por meio de regressão linear simples e múltipla, pelo método dos mínimos quadrados ordinários, cujos coeficientes, bem como seus indicadores de ajuste (critérios de seleção de modelos) são encontrados na Tabela 2.

Os coeficientes de determinação ajustados $\left(R^{2}\right.$ aj. $)$, obtidos pelo ajuste das equações com os modelos testados, podem ser considerados satisfatórios. Rossi et al. (2011) analisaram 10 modelos de relação hipsométrica para teca com idades entre 1; 5 e 26 anos, na região de Monte Dourado, no Estado do Pará, com $R^{2}$ aj. variando de 0,49 a 0,85 . Drescher (2004) testou modelos de relação hipsométrica para plantios de teca nos municípios de Brasnorte e Santo Antônio do Leverger, no Estado do Mato Grosso, com idades entre 8 e 14 anos, obtendo $R^{2}$ aj. de 0,53 a 0,90. Figueiredo (2005), testando um modelo hipsométrico genérico para povoamentos de teca de 8 e 10 anos, na região do Baixo Rio Acre, obteve valor de $R^{2}$ aj. igual a 0,72. Já Christo et al. (2012), estudando povoamentos de teca com idades entre 7 e 12 anos, na região de Alta Floresta, no Estado do Mato Grosso, observaram valores inferiores de $\mathrm{R}^{2}$ aj. para seis modelos de relação hipsométrica, variando de 0,41 a 0,60. Pereira et al. (2014), estudando povoamentos de 13 anos em Moju-PA, encontraram $R^{2}$ aj. de 0,45 a 0,82, ao testarem 11 modelos hipsométricos. Diante disso, pode- 
se afirmar que há uma variação grande nos valores de $\mathrm{R}^{2}$ aj. para modelos hipsométricos para teca, dependendo da natureza dos dados e dos modelos testados.

O erro-padrão da estimativa é um critério tradicionalmente usado na avaliação da qualidade de ajuste de equações em inventários florestais do meio florestal. Esse pode ser empregado nas dimensões absoluta ( $\left.S_{y x}\right)$ e percentual ( $\left.S_{y x} \%\right)$ (Sanquetta et al., 2014b).

Tabela 2 - Equações ajustadas e indicadores dos critérios de seleção de modelos para 400 indivíduos jovens de Tectona grandis em Redenção-PA. Adjusted equations and indicators of the selection criteria of the models for the 400 young individuals of Tectona grandis in Redenção-PA.

\begin{tabular}{lcccc}
\hline Equação & $\mathrm{R}^{2}$ aj. & $\mathrm{S}_{\mathrm{yx}} \%$ & $\mathrm{AIC}$ & $\mathrm{BIC}$ \\
\hline (1) $h t=\beta_{0}(1,104971)+\beta_{1}(0,676120) \mathrm{DAP}$ & 0,88 & 11,73 & 69,35 & 75,27 \\
(2) $h t=\beta_{0}(7,240803)+\beta_{1}(0,018222) \mathrm{DAP}{ }^{2}$ & 0,81 & 15,08 & 270,46 & 276,38 \\
$(3) h t=\beta_{0}(-21,715847)+\beta_{1}(12,153799) \ln (\mathrm{DAP})$ & 0,88 & 11,83 & 75,91 & 87,83 \\
$(4) h t=\beta_{0}(15,787633)+\beta_{1}(-0,926242) \mathrm{DAP}+\beta_{2}(0,042781) \mathrm{DAP}^{2}$ & 0,89 & 11,37 & 46,16 & 50,04 \\
$(5) \ln (h t)=\beta_{0}(25,294417)+\beta_{1}(-211,405255)(1 / \mathrm{DAP})$ & 0,81 & 15,03 & 267,37 & 273,29 \\
$(6) \ln (\mathrm{ht})=\beta_{0}(-21,715847)+\beta_{1}(27,985156) \log (\mathrm{DAP})$ & 0,88 & 11,83 & 75,91 & 81,84 \\
\hline
\end{tabular}

$\beta_{0}$ e $\beta_{1}$ - coeficientes estimados por regressão; $h t$ - altura total do fuste (m); DAP - diâmetro a altura do peito $(\mathrm{cm})$; $\mathrm{R}^{2}$ aj.: coeficiente de determinação ajustado; Syx\% erro-padrão da estimativa, em porcentagem; AIC: critério de informação de Akaike, e BIC: critério de informação Bayesiano.

O erro-padrão da estimativa é um critério tradicionalmente usado na avaliação da qualidade de ajuste de equações em inventários florestais do meio florestal. Esse pode ser empregado nas dimensões absoluta $\left(S_{y x}\right)$ e percentual ( $S_{y x} \%$ ) (Sanquetta et al., 2014b). O presente estudo considerou o erro-padrão da estimativa na dimensão percentual porque os modelos apresentam diferentes conformações matemáticas concernentes à variável dependente. Nesse sentido, calculou-se o erro-padrão da estimativa percentual para os seis modelos testados, os quais variaram de $11,37 \%$ a $15,08 \%$, sendo considerados, em princípio, satisfatórios. Rossi et al. (2011) encontraram erros-padrão da estimativa percentuais entre 11,94 e $54,34 \%$. Pereira et al. (2014) observaram que os erros-padrão da estimativa para os modelos testados pelos autores variaram de 16,7 a $18,7 \%$. No estudo realizado por Drescher (2004), o autor reportou valores de $S_{y x} \%$ da ordem de 4,9 a $16,9 \%$, dependendo do modelo testado. Figueiredo (2005) reportou erro de 7,32\%, enquanto Christo et al. (2012), por sua vez, encontraram valores de 11,9 a $54,4 \%$ em seu estudo. Portanto, constata-se que a magnitude do erro percentual das equações também varia consideravelmente e que é influenciada pelos dados utilizados no ajuste e pela formulação matemática dos modelos.

No tocante aos valores apresentados pelos critérios 3 e 4, não foram encontradas referências sobre ajuste de equações hipsométricas para a espécie na literatura. Os critérios AIC e BIC ainda são menos utilizados na avaliação da qualidade de ajuste de modelos de regressão, mas vêm sendo considerados por alguns autores (Burnham \& Anderson, 2002; Vismara, 2009) como mais adequados para esse fim. Porém, Sanquetta et al. (2014b) demonstraram que os critérios gerais de ajuste (incluindo AIC e BIC) podem conduzir a um lugar comum na seleção de modelos de regressão empregados em inventários florestais.
Levando-se em conta os critérios gerais de ajuste, pode-se verificar que os modelos tiveram comportamentos semelhantes e, a princípio, com indicadores satisfatórios em termos de ajuste, dado que os valores de $\mathrm{R}^{2}{ }_{\mathrm{aj}}$. e $\mathrm{S}_{\mathrm{yx}} \%$ foram próximos. Todavia, os valores de $\mathrm{AlC}_{c}$ e BIC apontam para diferenças relevantes entre os modelos. Os modelos (1), (3), (4) e (6) realmente tiveram desempenho parecidos, mas os modelos (2) e (5) foram inferiores em relação aos critérios gerais de seleção de modelos.

A análise dos valores estimados sobre a nuvem de pontos (Figura 1) e a análise da dispersão dos resíduos (Figura 2) indicaram que o modelo (2) é tendencioso, uma vez que sua formulação matemática denota uma relação quadrática entre o DAP e ht, o que não encontra fundamento nos dados reais. Os modelos (3) e (5), por sua vez, demonstram vieses em subestimar sistematicamente a altura a partir do DAP $15 \mathrm{~cm}$. Os modelos (1) e (4) apresentam comportamentos semelhantes em termos de dispersão de resíduos, e o modelo (6) tende a apresentar superestimavas para árvores muito pequenas (valores baixos de DAP).

Dos modelos avaliados, os modelos (1) e (3) apresentaram 3 valores de resíduos abaixo dos $-100 \%$, chegando até a $-131 \%$ e até a $-300 \%$, respectivamente. Já o modelo (2) apresentou 2 valores de resíduos inferiores aos $-100 \%$, chegando até a $-300 \%$. Porém, por razões de escala, esses valores não estão apresentados nos gráficos da Figura 2.

Considerando-se a distribuição dos resíduos, pode-se perceber que os modelos (1) e (4) apresentam ajustes satisfatórios. O modelo (4) demonstrou leve superioridade em relação ao modelo (1), porque o traçado curvilíneo de sua conformação matemática se adere melhor ao formato da nuvem de pontos, sendo selecionado neste estudo como o mais indicado para o conjunto de dados em análise. Todavia, há que se fazer a ressalva de que o modelo (4) tem conformação parabólica, o que pode sugerir 
decréscimo de ht com o aumento do DAP a partir de determinado valor. Para os ajustes realizados neste estudo, as estimativas devem ficar limitadas ao espectro dos dados empregados no trabalho, ou seja, até $22 \mathrm{~cm}$ de DAP. A partir desse valor, as estimativas são extrapolações, as quais podem gerar valores anômalos, particularmente a partir de $38 \mathrm{~cm}$ de DAP, quando o efeito parábola se manifesta no modelo de Trorey no presente caso, gerando alturas que vão diminuindo com o aumento do DAP, convertendo-se em valores negativos a partir de $80 \mathrm{~cm}$ de DAP, o que não condiz com a realidade, uma vez que não existem alturas negativas. Portanto, o modelo (4) pode ser seguramente empregado, gerando estimativa de qualidade satisfatória até $22 \mathrm{~cm}$ de DAP.

Com os 35 dados advindos de medição de árvores derrubadas, cujas alturas reais foram medidas diretamente com trena, efetuou-se a validação da equação de Trorey ajustada para as 400 árvores. Os resultados da validação pelos testes de Qui-quadrado e t-pareado demonstraram que não existe diferença significativa entre os valores reais e estimados pela equação de Trorey $(\alpha=0,05)$. Os valores estimados aproximam-se dos valores reais e houve pequena dispersão dos resíduos, ficando os mesmos confinados próximos à linha de regressão. Portanto, fica demonstrado que a equação de Trorey ajustada é satisfatória para as condições vigentes neste estudo.

\section{Conclusões}

Os seis modelos testados para relação hipsométrica de povoamentos jovens de teca mostraram, a princípio, indicadores gerais de ajuste considerados satisfatórios, mas a análise gráfica de resíduos evidenciou que quatro deles apresentam vieses nas estimativas.

Os modelos da reta e de Trorey não apresentaram vieses relevantes nas estimativas, mas o segundo foi selecionado como o mais preciso e acurado, considerando os critérios gerais de ajuste e a análise da distribuição dos resíduos.

A equação gerada pelo modelo de Trorey foi validada com dados independentes e demonstrou propiciar estimativas confiáveis e sem viés, considerando as condições vigentes neste estudo.

A hipótese testada neste trabalho pode ser aceita, uma vez que a relação entre ht e DAP, em indivíduos jovens de teca, é forte o suficiente para possibilitar o desenvolvimento de equações hipsométricas satisfatórias para plantios jovens.
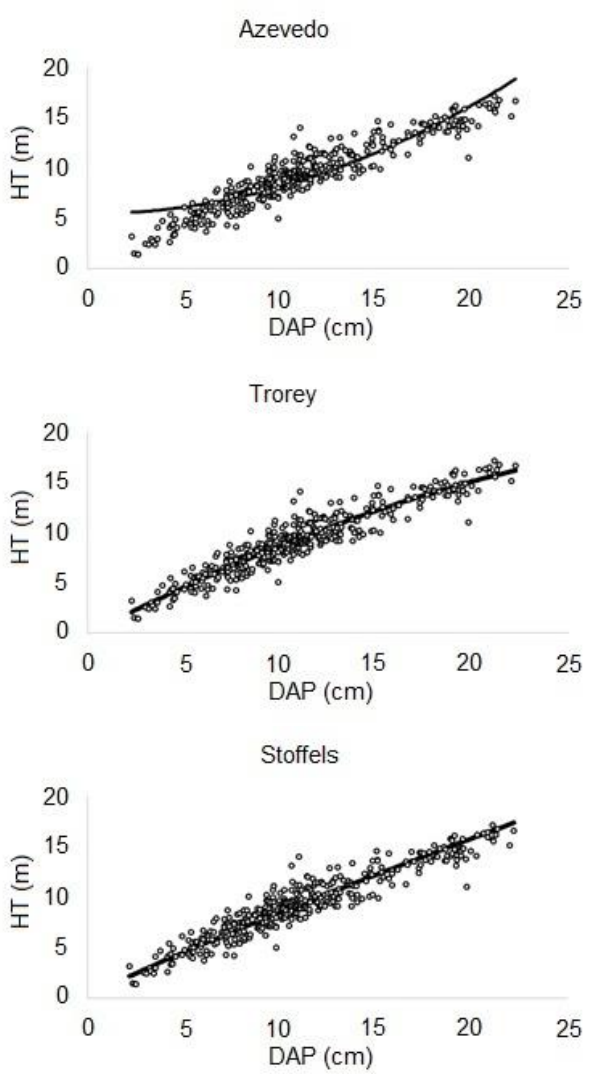

Figura 1 - Linhas de ajuste de equações hipsométricas ajustadas para 400 pares de valores de DAP e ht de indivíduos jovens de Teca (Tectona grandis) em Redenção-PA. Lines of adjustment of the hypsometric equations for the 400 pairs of values of DAP and ht of young individuals of Teca (Tectona grandis) in Redenção-PA. 
Reta

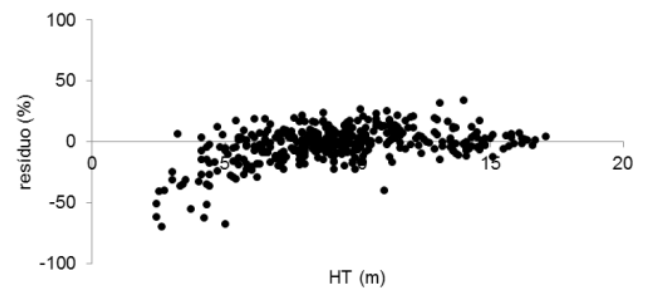

Henricksen

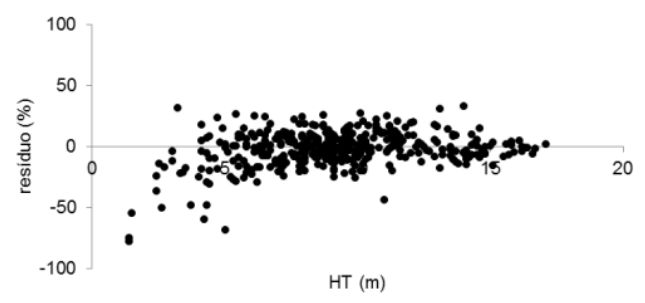

Curtis

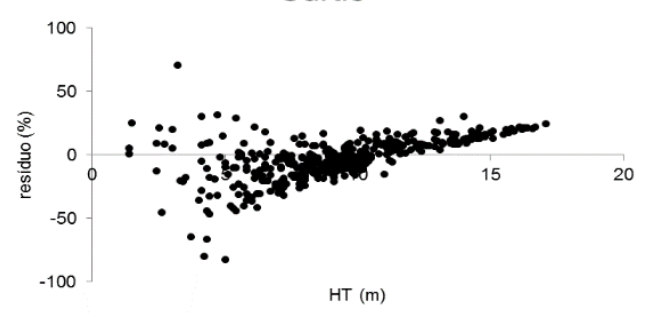

Azevedo

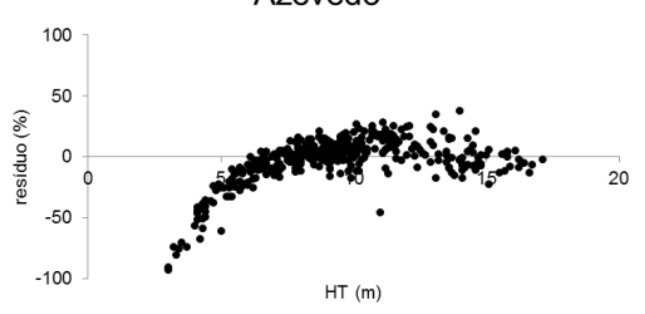

Trorey

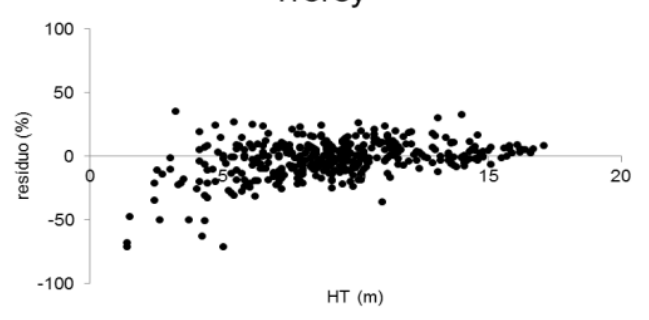

Stoffels

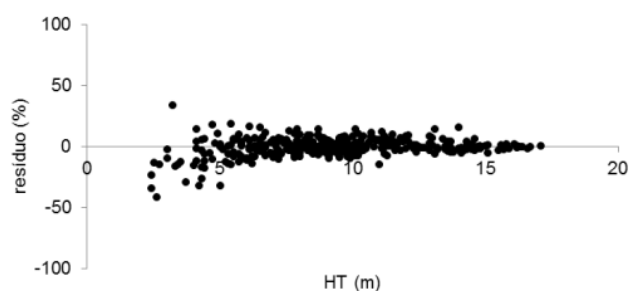

Figura 2 - Distribuição dos resíduos de seis equações hipsométricas ajustadas para 400 indivíduos jovens de Teca (Tectona grandis) em Redenção-PA. Distribution of residuals of six hypsometric equations adjusted for 400 youg individuals of Teak (Tectona grandis) in Redenção-PA.

(a) Trorey - validação

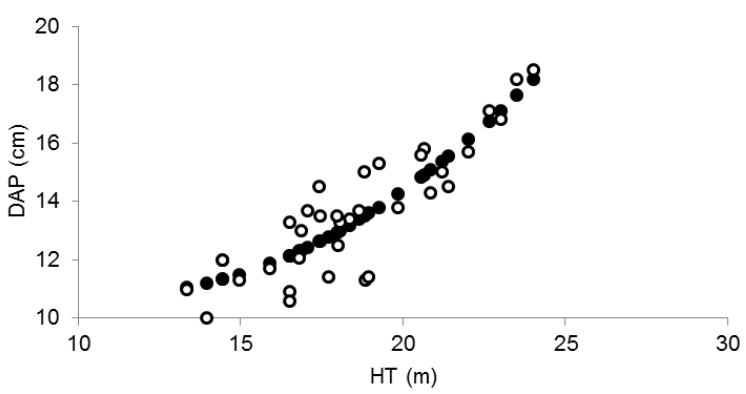

(b) Trorey - validação

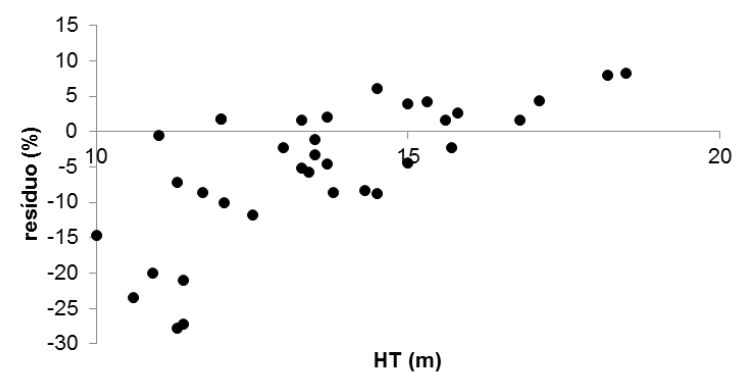

Figura 3 - Equação ajustada com o modelo de Trorey (a) versus dados reais de 35 árvores com medidas tomadas com trena e utilizadas para a validação da mesma e respectiva distribuição dos resíduos (b) para indivíduos jovens de Tectona grandis em Redenção-PA. Adjusted equation with the Trorey's model (a) versus real data (b) of 35 trees measured by measuring tape and used for validation distribution of residuals for young individuals of Tectona grandis in Redenção-PA.

\section{Referências}

Akaike $H$ (1973) Information theory as an extension of the maximum likelihood principle. In: Second international symposium on information theory. Budapest: Akademiai Kiado. p. 267-281.

Azevedo CP, Muroya K, Garcia IC, Lima RMB, Moura JB, Neves EJM (1999) Relação hipsométrica para quatro espécies florestais em plantio homogêneo e em diferentes idades na Amazônia ocidental. Boletim de Pesquisa Florestal 39:5-29.
Bhat KM (2000) Timber quality of teak from managed tropical plantations with special reference to Indian plantations. Bois et Forêts des Tropiques 263(1):5-29.

Burnham KP, Anderson DR (2002) Model selection and multimodel inference: a practical informationtheoretic approach, 2 edn, Springer. 488p.

Christo JC, Dias NA, Figueiredo Filho A, Menon FV (2012) Modelagem e crescimento de Tectona grandis Linn F. no município de Alta Floresta - MT a partir de anatro (análise de tronco) completa. In: Congresso Florestal Paranaense. Anais... p. 01-09. 
Curtis RO (1967) Height diameter and height age equations for second growth Douglas-fir. Forest Science 13(4):365-375.

Drescher R (2004) Crescimento e produção de Tectona grandis Linn F., em povoamentos jovens de duas regiões do Estado do Mato Grosso - Brasil. 2004. $116 \mathrm{f}$. UFSM (Tese de doutorado em Engenharia Florestal).

FAO (2012) Teak resources and market assessment 2010. FAO. 42 p.

Figueiredo EO (2005) Avaliação de povoamentos de teca (Tectona grandis L.F.) na microrregião do Baixo Rio Acre. UFLA. (Dissertação de Mestrado em Engenharia Florestal).

Henricksen HA (1950) Height-diameter curve with logarithmic diameter: brief report on a more reliable method of height determination from height curves, introduced by the State Forest Research Branch. Dansk Skovforen Tidsskr 35:193-202.

Hoare P, Patanapongsa N (1988) Long-rotation, high value trees: an alternative strategy for private forestry. Commonwealth Forestry Review 4(67):351-361.

IDESP (2012) Estatística Municipal de Redenção. Prefeitura municipal de redenção. 44p.

Keogh R, Fallas J, Mora F (1978) Teca (Tectona grandis) en Costa Rica. FAO. 19p.

Leite HG, Oliveira-Neto RR, Monte MA, Fardin L, Alcantara AM, Binoti LMS, Castro RVO (2011) Modelo de afilamento de cerne de Tectona grandis L.f. Scientia Forestalis 39(89):53-59.

Monteuuis O, Goh DKS (1999) About the use of clones in teak. Bois et Forêts des Tropiques. 261(3):28-37.

Pérez Cordero LD, Kanninen M (2003) Heartwood, sapwood and bark content, and wood dry density of young and mature teak (Tectona grandis) Trees Grown in Costa Rica. Silva Fennica 37(1):45-54.

Pereira KD, Paixão MVC, Monteiro CWB, Laurido FT, Ferreira PST (2014) Ajuste de modelos hipsométricos para árvores de Tectona grandis L.f. no município de Mojú, Pará. Enciclopédia Biosfera 10(18):181-189.
Rossi AS, Drescher R, Pelissari, AL, lanssanova IR (2011) Relação hipsométrica e crescimento de Tectona grandis L.f. no município de Monte Dourado, Pará. Scientia Forestalis 39(91): 301-307.

Sanquetta MNI, Sanquetta CR, Corte APD, Mognon F, Behling A (2014a) Incremento diamétrico e percentuais de cerne e de casca em povoamentos de Tectona grandis L.f. no sudeste do Pará. Enciclopédia Biosfera 10(18):1946-1954.

Sanquetta CR, Dalla Corte AP, Behling A, Piva IRO, Sanquetta MNI (2014b) Uso de critérios estatísticos de seleção de modelos alométricos para estimar biomassa individual de espécies. In: Dalla Corte AP, Sanquetta CR, Rodrigues AL, Machado AS, Péllico Netto S, Figueiredo Filho A, Nogueira GS (ed) Atualidades em Mensuração Florestal. UFPR. p.398402.

Schmidt PB (1977) Determinação indireta da relação hipsométrica para povoamentos de Pinus taeda L. Floresta 8(1):24-27.

Schwarz G (1978) Estimating the dimensions of a model. Annals of Statistics 6:461-464.

Stoffels A, Soest JV (1953) The main problems in sample plots. Ned Bosbouwtijdschr 25:190-199.

Tonini H, Costa MCG, Schwengber IAM (2009) Crescimento da teca (Tectona grandis) em Reflorestamento na Amazônia Setentrional. Pesquisa Florestal Brasileira 59:05-14.

Trorey LGA (1932) A mathematical method for construction of diameter-height curves based on site. Forest Chronicle 8(2):121-132.

Tze WTY (1999) Recovery and quality of lumber from mature teak (Tectona grandis) planted in Sabah, Malaysia. Journal of Tropical Forest Products 5(2):115-123.

Vismara ES (2009) Mensuração da biomassa e construção de modelos para construção de equações de biomassa. USP (Dissertação de Mestrado em Recursos Florestais: Silvicultura e Manejo Florestal). 